Accretion Phenomena and Related Outflows, IAU Colloquium 163

ASP Conference Series, Vol. 121, 1997

D.T. Wickramasinghe, L. Ferrario, and G.V. Bicknell, eds.

\title{
Planetary Formation in Protostellar Disks
}

\author{
D.N.C. Lin
}

\section{Lick Observatory, University of California, Santa Cruz, CA 95064}

\begin{abstract}
Recent discoveries of planets around other stars suggest that planets are ubiquitous and their dynamical properties are diverse. We reviewed the formation mechanism for protoplanets and the post-formation planet-disk tidal interaction which may have led the short-period planets to their present configuration. We suggest that these planets may be the survivors of a populations of similar planets which have plunged into and contaminated the stellar convection zone. In the context of the solar system, the mass of the giant planets and the present distribution of the minor planets may be used to infer the structure and evolution for the primordial solar nebula. The large eccentricity of 70 Vir and HD 114762 may be due to cohesive collisions in planetary systems which become unstable during their long term orbital evolution.
\end{abstract}

\section{Introduction}

The hypothesis that the planets in our solar system were formed in a flattened gaseous disk was proposed to account for the origin of their present dynamical properties. Recent observations indicate that typical star-forming dense cores have specific angular momentum $>10^{21} \mathrm{~cm}^{2} \mathrm{~s}^{-1}$ (Goodman et al 1993) such that their collapse leads to rotationally supported disks analogous to the primordial solar nebula (Terebey et al. 1984). During its collapse, gas around one young stellar objects (YSO), HL Tau, is observed to rotate (Sargent \& Beckwith 1987, Hayashi et al. 1993) with an adequate amount of angular momentum to form a 50-100 AU disk (Lin et al. 1993). This inner disk has been resolved (Lay et al. 1994, Mundy et al. 1996).

In unresolved classical T Tauri stars (CTTS), the presence of AU-size disks is inferred from the spectral energy distribution over more than 2 orders of magnitude in the IR wavelength. The observed power index is consistent with that expected from either the viscous dissipation of accretions disks with a steady mass transfer rate $\dot{M} \sim 10^{-7} M_{\odot} \mathrm{yr}^{-1}$, or the reprocessed stellar radiation emitted by circumstellar geometrically-thin opaque disks (Adams et al. 1987).

Between 25 to $75 \%$ of YSOs in the Orion nebula appear to have disks (Prosser et al. 1994, McCaughrean \& Stauffer 1994). In the Taurus-Auriga complex, more than half the T Tauri stars are weak-line systems (WTTS) with little or no IR excess and estimated age $\tau_{*} \sim 10^{6-7} \mathrm{yr}$ (Walter et al. 1988). Among open clusters, the relative abundance of WTTS is correlated with the cluster's age (Lada et al. 1993). The disappearance of IR excess around WTTS suggests that either dust grains have coagulated and settled or the gas is mostly 
vanished in the disk (Skinner et al. 1991, Strom et al. 1993). The latter interpretation is supported by the observed depletion of $\mathrm{CO}$ around YSOs with ages of a few $10^{6}$ yrs (Zuckerman et al. 1995).

The frequent sightings of protostellar disks around YSOs, with properties similar to the solar nebula, suggest the necessary condition for planetary formation is generally satisfied. If their formation is a robust process, planets would be ubiquitous in nature. The discovery (Mayor \& Queloz 1995, Marcy et al. 1996) of a short-period ( $P=4.23$ days) negligible-eccentricity $(e=0)$, low-mass ( $M_{p}=0.5 M_{J} / \sin i$ where $M_{J}$ is Jupiter's mass and $i$ is the inclination of the system's orbit with the line of sight) companion to 51 Peg marks a milestone in the search for extrasolar planetary systems. Similar short-period (type II) planets were subsequently found around $55 \rho$ Cnc (Butler, Marcy, \& Williams 1996), $\tau$ Boo, and $v$ And with $e=0, P=14.65,3.31,4.6$ days, and $M_{p}=0.78$, 3.87 , and $0.6 M_{J} / \sin i$ respectively. In $55 \rho \mathrm{Cnc}$ and $\tau$ Boo, there are indications of additional planets with several year periods (Butler et al. 1996). There are also eccentric (type III) planets around HD 114762 (Latham et al. 1989), 70 Vir (Marcy \& Butler 1996), and 16 Cyg (Cochran et al 1996) which have orbital semi major axes $(a=0.34,0.43$, and $1.5 \mathrm{AU}), e(=0.35,0.38$, and 0.6$)$, and $M_{p}$ $\left(=9,6.6\right.$, and $\left.1.7 M_{J} / \sin i\right) .47 \mathrm{UMa}$ has a planet with $a(2.1 \mathrm{~A} . \mathrm{U}),. e(0.03)$, and $M_{p}\left(2.39 M_{J}\right)$ similar to the Jovian (type I) planets (Butler \& Marcy 1996).

\section{Protoplanetary formation and migration}

According to the conventional theories (Pollack et al. 1996), the first stage of protoplanetary formation is the rapid build up of solid cores through the coagulation of solid planetesimals (Safronov 1969, Wetherill \& Stewart, 1989). After acquiring a fraction of an Earth mass $\left(M_{\oplus}\right)$, these cores begin to accret gas from the solar nebula and to attain a quasi static atmosphere which is heated by the bombardment of solid particles onto the cores. When the cores' mass $M_{\text {core }}$ increases above a critical value $\left(M_{\text {crit }} \sim\right.$ a few $\left.M_{\oplus}\right)$, the planetary atmosphere becomes unstable, undergoes collapse, and the cores dynamically accret the gas (M1zuno 1980, Bodenheimer \& Pollack 1986).

The accretion rate $(\dot{M})$ increase rapidly with $M_{p}$. For a planet with $M_{p} \sim$ $M_{J}, \dot{M} \sim 10^{-6} M_{\odot} \mathrm{yr}^{-1}$. During this phase of rapid growth, the protoplanet also exerts a tidal perturbation on the disk (Papaloizou \& Lin 1984). In the limit that the disk has a relatively low viscosity $(\nu)$, the protoplanet induces the formation of a gap in the disk near its orbit when its Roche radius $\left(\left(M_{p} / 3 M_{*}\right)^{1 / 3} a\right.$ where $M_{*}$ is the mass of the central star) increases beyond the disk thickness $H$ (Lin \& Papaloizou 1993). After the gap formation, $M_{p}$ no longer increases since the disk gas cannot reach the protoplanet. At $a<0.1 \mathrm{AU}$, the acquisition of $1 M_{J}$ requires $H \sim 0.1 a$ and a midplane temperature $\left(T_{c}\right)>2 \times 10^{3} \mathrm{~K}$. Since refractory material cannot condense at such high $T_{c}$ (Palme \& Boynton 1993), the short-period planets are unlikely to be formed in situ.

We propose that all three type planets were formed several $\mathrm{AU}$ away from their host stars through the processes outlined in the standard scenario (Lin et al. 1996). After the termination of their growth through gap formation, protoplanets continue to tidally remove/supply angular momentum from/to the disk interior/exterior to the gap (Goldreich \& Tremaine 1980, Lin \& Papaloizou 
$1986)$. Since gas flow across the gap is quenched, both the surface density $(\Sigma)$ and the flux of tidal transfer of angular momentum $\left(\dot{J}_{t}\right)$ interior to the gap decrease as gas diffuses toward the star. In the outer disk, $\Sigma$ and $\dot{J}_{t}$ maintain their value as gas accumulates due to the prevention of the inward viscous diffusion by the protoplanet's tidal torque. The imbalance of $\dot{J}_{t}$ between the inner and outer disk leads to the inward orbital migration of the protoplanet. If $\Sigma>>M_{p} / a^{2}$, the orbital migration of the protoplanet would be coupled to the viscous evolution of the disk (Lin \& Papaloizou 1986, Takeuchi et al. 1996). In this limit, $\Sigma$ would adjust until the viscous angular momentum flux $\left(\dot{J}_{\nu}\right)$ becomes comparable to $\dot{J}_{t}$ such that the disk would evolve and protoplanet would migrate on the viscous diffusion timescale $\left(\tau_{\nu}\right)$ at the radius $\left(r_{\nu}\right)$ of maximum stress (approximately where most of the disk mass is contained). In the solar nebula, $r_{\nu} \sim$ a few AU. If the fading of the IR excess in protostellar disks is due to their viscous evolution, $\tau_{\nu} \sim \tau_{*} \sim 10^{6} \mathrm{yr}$.

\section{YSO-protoplanet-disk interaction}

\subsection{The interruption of protoplanetary migration.}

A protoplanet's migration is terminated in the proximity of the stellar surface under at least two conditions. 1) The spin period $\left(P_{*}\right)$ of CTTS is observed to cluster around 8 days (Bouvier et al. 1993) despite the accretion of rapidly rotating disk gas. This spin rate may be attained if the CTTS have a strong $\left(>10^{3}\right.$ gauss) stellar magnetic field which would induce a) the truncation of the inner region of the disk to the magnetosphere radius $\left(r_{m} \sim\right.$ a few $\left.10^{11} \mathrm{~cm}\right)$ and b) the synchronization of $P_{*}$ with the Keplerian period of the disk gas at $r_{m}\left(P_{k}\right)$ (Konigl 1991). After migrating into this cavity, the protoplanet interacts with the disk through its low order main motion resonances such that $\dot{J}_{t}$ is reduced by a factor of $\sim M_{p} / M_{*}$ (Goldreich \& Tremaine 1978). Interior to the $2: 1$ resonance of the gas at $r_{m}$ (at $a<0.63 r_{m}$ ), the protoplanet is decoupled to the disk and its migration is interrupted. We note that the periods of several planets $\left(P_{p}\right)$ are nearly half the observed peak in $P_{*}$ (Lin et al. 1996).

2) In the limit of a weak stellar magnetic field, the disk extends to the proximity of the stellar surface at $r_{*}$. The accretion of disk gas leads to a rapid stellar rotation and a small corotation radius, $r_{c}$ (Kley \& Lin 1996). Outside $r_{c}$, star-planet tidal friction induced angular momentum transfer from the star's spin to the planet's orbital motion at a rate $\left(\dot{J}_{*}\right)$ which is a rapidly increasing function of $a$ (Goldreich \& Soter 1966). For the range of the dissipation parameter $\left(Q \simeq 10^{5}\right)$ appropriate for YSOs, an equilibrium is established with $\dot{J}_{*} \simeq \dot{J}_{t}$ at a radius $r_{e} \sim$ a few times $r_{*}$ which is comparable to or less than $a$ of the short period planets (Lin et al. 1996).

The star-planet tidal interaction is also important inside the magnetosphere cavity in the first stoppage scenario. Since $r_{c} \sim r_{m}$, a protoplanet in the cavity have $a<r_{c}$ so that it spirals inward as it loses the angular momentum to the star's spin. But for a sufficiently strong field, $r_{m}$ is relatively large so that the planet's orbit decays on a timescale $\left(\tau_{d}\right)$ longer than the YSO contraction timescale $\left(\tau_{y}\right)$. During the post $\mathrm{T}$ Tauri contraction, the tidal interaction weakens and the protoplanet survives. For intermediate field strength, 1) $r_{e}<r_{m}$ 
so that the protoplanet enters the magnetosphere cavity before attaining a tidal equilibrium but 2) $\tau_{d}\left(r_{m}\right)<\tau_{y}$ and the protoplanet spirals into the YSO before it contracts onto the main sequence.

In the weak field limit, $r_{m}<r_{e}$ and the protoplanet migration is temporarily interrupted at $r_{e}$. But, $P_{*}$ decreases and $r_{c}$ increases as angular momentum is drained from the YSO's spin on a timescale $\tau_{\dot{p}} \sim \tau_{\nu} M_{*} j_{*} / M_{d} j\left(r_{\nu}\right)$. The specific angular momentum $\left(j\left(r_{\nu}\right)\right)$ of the disk gas at $r_{\nu}$ is $\sim 10^{2}$ times larger than that $\left(j_{*}\right)$ in the YSO's envelope. For relatively massive $\left(M_{d}>0.1 M_{*}\right)$ disks $\tau_{\dot{p}}<\tau_{y}$ and the protoplanet resumes its inward migration into the YSO after $r_{c}$ becomes larger than $r_{e}$. The inner boundary of the accretion disk is extended to $r_{*}$ or $r_{m}$ if $r_{m}>r_{*}$ and the YSO spins up to corotate with the gas at that location.

\subsection{Multiple short-period planetary systems.}

Significant orbital migration of protoplanets with $M_{p}>M_{J}$ requires the substantial amount of residual disk gas (with $M_{d} \sim 0.1 M_{\odot}$ ) out of which, additional protoplanets may be formed. This expectation is consistent with the suggestive detections of multiple planets around $55 \rho \mathrm{Cnc}$ and $\tau$ Boo. Provided the solid particles has a surface density $\left(\Sigma_{p}\right)$ which decreases with $a$ (Hayashi et al. 1985), the growth timescale of protoplanetary cores $\left(\tau_{\text {grow }}\right)$ increases with $a$. After the first born protoplanet emerges at $a_{1}$ and migrates to a few $r_{*}$, additional protoplanets may be formed with $a>a_{1}$, migrate inward, and sweep up the gas and protoplanetary cores ahead of their inward path, though their Lindblad resonances.

As the later arrivers approach to the magnetosphere cavity (in scenario 1) the sweep up gas flows onto the YSO along the field lines while they capture the earlier arrived protoplanets onto their Lindblad resonances and induce them to resume their inward migration. This locked migration is again interrupted when the later arrivers enter into magnetosphere cavity. After this adjustment, the closest protoplanet would perish into the YSO if its $\tau_{\dot{p}}\left\langle\tau_{y}\right.$. But if $r_{m} \gg r_{*}$, the locked migration would lead to a system of commensurable planets.

If the inward migration of the first born protoplanet is interrupted by the YSO's tidal barrier (scenario 2), $\Sigma$ in the swept-up ring would adjust until a quasi equilibrium is established in which angular momentum is being transfered at a constant rate from the YSO to the innermost protoplanet then to the trapped ring and the next arriver and eventually to the outer disk. In this configuration, the protoplanets become the shepherds to a trapped ring, analogous to Saturn's F ring. But as the YSO loses its angular momentum, the innermost protoplanet resumes its inward migration when its $r_{e}<r_{c}$. Subsequently, angular momentum is tidally transfered from the innermost protoplanet to the YSO. After the innermost protoplanet plunges into the YSO, $r_{c} \sim r_{e}$ for the next protoplanet. The consumption of the protoplanet by the YSO continues to recur on the timescale $\sim \tau_{\dot{p}}$ until gas in the disk is sufficiently depleted.

The depletion of the disk gas leads to a reduction in both $\dot{J}_{t} \sim \dot{J}_{\nu}$ and $\dot{M}$ and provides the necessary condition for the YSO to undergo contraction onto the main sequence. In the strong field limit (scenario 1), $r_{m}$ is unlikely to decrease significantly despite the reduction in $r_{*}$ such that the short-period protoplanets survive their tidal interaction with the YSOs. In the weak field limit (scenario 2), if $M_{d}<0.1 M_{*}, \tau_{\dot{p}}>\tau_{y}$ and the YSO would spin up during its 
contraction. If the disk depletes faster than the star contracts, $r_{e}$ would increase slightly leading to a slight expansion of the planet's orbit. Since $r_{c}<r_{e}$ for all the planets, they would all be held back by the YSO's tidal barrier.

\section{Formation of the solar system.}

The formation of Jovian planets is proceeded by the acquistation of $10-40 M_{\oplus}$ core in a gaseous environment. For a population of planetesimals, $\tau_{\text {grow }}$ increases with their velocity dispersion $(\sigma)$ and is inversely proportional to $\Sigma_{p}$ (Safronov 1969). Under the optimum condition of low $\sigma, \tau_{\text {grow }} \sim \tau_{*} \sim 10^{6}$ yr if $\Sigma_{p}$ is at least four times (Lissauer 1987) that $\left(\Sigma_{\min }\right)$ inferred from the minimum-mass hypothesis (Cameron 1973, Hayashi 1981). The corresponding $M_{p} \sim 0.1 M_{\odot}$ is comparable to that estimated for some CTTS (Beckwith et al. 1992). High mass disks are also inferred for YSOs which undergo FU Orionis outbursts (Herbig 1977) during which $\sim 10^{-2} M_{\odot}$ is accreted onto the central stars over $\sim 10^{2} \mathrm{yr}$ duration (Hartmann et al. 1993).

The observed rotation of molecular-cloud cores and HL Tau's infalling envelope suggests that most infalling gas arrives onto the disk before joining the star (Tereby et al. 1984). During the protracted infall stage, a fraction of the heavy elements condenses and sediments in the disk such that growth of planetesimals is a continuous process. The accumulation of the solid particles enhances the disk's metallicity above the solar value so that $\tau_{\text {grow }}$ is reduced to $<10^{6} \mathrm{yr}$ in a gravitationally stable disk.

In minimum mass solar nebula model, $\Sigma_{p}$ is a decreasing function of the disk radius $r$ (Hayashi et al. 1985). Despite the enhancement in $\Sigma_{p}$ by the condensation of volatile (such as ice) grains, $\tau_{\text {grow }}$ is likely to be an increasing function of $r$, In systems with a large $\Sigma_{p}$, Jupiter's formation may be proceeded by the emergence of several protoplanetary cores, with $M_{\text {core }}>M_{\text {crit }}$, and protogiant planets in the inner solar system where the terrestrail planets reside today. We propose that these protoplanets perished in their infancy as a consequence of protoplanetary migration (Lin 1996). The self regulated protoplanetary formation and migration would persist in the solar nebula as long as it contains $\Sigma>\Sigma_{\min }$ for a duration $>\tau_{\text {grow }}$. For smaller value of $\Sigma$, the protoplanetary disk can no longer produce any additional planets or to influence the evolution of those remaining.

In this scenario, Jupiter and Saturn are the last survivors of a planet formation and destruction process that operated on a timescale comparable to $\tau_{\nu}$. Through resonance forcing, the migrating protogiant planets induce the planetesimals and protoplanetary cores along their path to migrate with them so that the inner solar system is cleared out repeatedly. The residual nebula gas diffuses to fill the region vacated by the retreating protogiant planets. Subsequent sublimation and condensation of grains provide a fresh supply of solid material to rebuild the planetesimal population. The terrestrial planets such as the Earth is the byproduct of the late stage evolution. In contrast, the first generation planetesimals formed beyond the orbit of Jupiter are preserved. The outward migration of Neptune may have led to the capture of Pluto and several other Kuiper belt objects into their observed commensurable configuration (Lin 1986, Malhotra 1995). 


\section{Planetary orbital evolution around main sequence stars}

\subsection{The demise of short-period planets}

The observed correlation between their $P_{*}$ and age indicates that YSOs spin up during their contraction and main sequence stars spin down as angular momentum is lost through winds (MacGregor \& Brenner 1991). In three short period planets, $r_{c}>a$ such that angular momentum is being transfered from their orbit to the central star. These planets resume their inward migration on the timescale $\tau_{d}>\tau_{m s}$ the main sequence lifespan of their host stars (Rasio $e t$ al 1996). The planet with the smallest $\tau_{d}$ is around $\tau$ Boo and its $P_{p}$ appears to be tidally locked with the stellar $P_{*}$ (Butler et al. 1996). In this system, the moment of inertia in the stellar envelope is less than that in the planet's orbit such that the loss of angular momentum via stellar winds would lead to the synchronization of $P_{*}$ and $P_{p}$.

The radiative envelope of YSOs with $M_{*}>$ a few $M_{\odot}$ may have a weak stellar field and relatively large $Q$ values such that they cannot easily interupt the protoplanets' inward migration. Although the extensive convective envelope of low-mass YSOs provided favorable conditions for the stopage of migrating protoplanets, it is also maintained during their main sequence evolution to provide relatively low $\mathrm{Q}$ values and short $\tau_{d}$. These selection effects may account the association of short period planets with only $\mathrm{G}$ and $\mathrm{F}$ dwarfs.

In $\S 3.2$, we speculated on the possibility that several protoplanets may have migrated to the proximity of the YSO's surface. Any planets once located interior to the orbits of the short-period planets would have migrated into the their host star by now. As the planets approach the host stars, they are exposed to the intense stellar radiation and wind. Around late type dwarfs, this heating effect is unlikely to induce a significant mass loss from planets with $M_{p}>M_{J}$. Nevertheless, the possibility remains that less massive planets may loss a significant amount of its mass. If a planet survives its migration and eventually become engulfed by the host star, it would be disrupted by tidal breakup, heating, and ram pressure stripping by ambient gas in the stellar envelope.

Supporting evidences for past planet demise may be found in the super solar metal abundance of $51 \mathrm{Peg}(\mathrm{G} 2 \mathrm{~V}), 55 \rho \mathrm{Cnc}(\mathrm{G} 8 \mathrm{~V})$, and $\tau$ Boo (F7V) (Butler et al. 1996). The convective envelope for each of these stars contains a few $10^{-2} M_{\odot}$. The demise of planets with $10-40 M_{\oplus}$ solid cores of heavy elements within the stellar envelope would lead to a significant metal enrichment there. Since the depth of the convection zone of a main sequence star decreases significantly with increasing $M_{*}$, the routine engulfing of planets by their hosts might lead to a tendency for hotter planetary hosts to show a general overall metallicity enhancement with respect to cooler ones.

Although protoplanets may also plunge into the mostly convective envelopes of YSOs, their contamination would not be noticeable. But, the demise of shortperiod planets during the main sequence evolution of their host stars implies that more of them may be found around WTTS. A search for short-period planets around WTTS can provide a useful test for the planet plunging scenario.

During their post main sequence evolution, the expansion of the host stars' envelope would engulf the observed short period planets. But, the convective envelope along the subgiant branch has a relatively large mass which would 
dilute the contamination due to the plunging planets. The debris of disrupted planets may be more conspicuous in relatively massive subgiants which have less massive convective envelopes.

\subsection{Long term stability and the formation of eccentric planets.}

In a relatively massive disk, several giant planets can be formed with $M_{p} \sim$ a few $(1-3) M_{J}$ and $a>1$ AU. Before the depletion of the disk gas (within $\left.10^{6-7} \mathrm{yr}\right)$, the protoplanet system is stable. But, after the depletion of the disk gas, mutual gravitational perturbation between the planets induces a gradual increase in their $e$ 's until their orbits become unstable and begin to cross each other on a timescale $\tau_{x}$. Based on the extrapolation of existing numerical results (e.g. Franklin et al. 1990, Chambers et al. 1996), $\tau_{x} \sim 10^{12-18} \mathrm{yr}$ for the solar system. But $\tau_{x}$ would reduce to $\sim 10^{8-12} \mathrm{yr}$ or $\sim 10^{5-8} \mathrm{yr}$ if all the Jovian planets have masses of $1 M_{J}$ or $2 M_{J}$ respectively.

We suggest that the massive eccentric planets may have acquired their orbital properties as a consequence of orbit crossing (Lin \& Ida 1996). Our numerical simulations indicate that systems with relatively massive planets and modest orbital separation are indeed unstable to orbit crossing. After these systems become unstable the planets undergo close encounters which excite a recoil speed comparable to their surface escape velocity $\left(V_{\text {esc }}\right)$. In the relatively deep potential of the inner solar system, the corresponding $e$ is limited to modest values (up to $\sim 0.6-0.7$ ). Thus, the seed planets remain in the inner regions, undergo repeated close encounters and eventually physical collisions. In the outer regions ( $a>10 \mathrm{AU}$ ), however, the Keplerain speed is $<10 \mathrm{~km} \mathrm{~s}^{-1}<V_{\text {esc }}$ so that close encounters between planets can significantly modify their $a$ 's and $e$ 's. A few close encounters can lead to the ejection of these planets with large $a$ 's such that they become dynamically isolated or gravitationally unbound. Cohesive collisions do not occur among the outer planets.

The merged bodies undergo minor orbital decay due to the gravitational perturbation by the outer planets and the dissipation of the colliding planets' relative kinetic energy. Afterward, the long term perturbation would reduce their $a$ (to $\simeq 0.5-1 \mathrm{AU}$ ) and preserve their $e \simeq 0.2-0.9$ such that the merged body becomes dynamically isolated from the others and merging events rarely recur thereafter. The orbital properties of the merged body are consistent with those of the eccentric planets around $70 \mathrm{Vir}$ and HD 114762.

Alternatively, large $e$ may be excited by the tidal interaction between the protoplanetary disk and relatively massive $\left(>10^{-2} M_{\odot}\right)$ companions. In this limit, the gap may be sufficiently wide to exclude the e-damping contribution from corotation resonances and large $e$ may also be excited by the tidal interaction through Lindblad resonances (Goldreich \& Tremaine 1979, 1980, Artymowicz 1993, Lin \& Papaloizou 1993, Artymowicz \& Lubow 1996). The large radial excursion associated with a large $e$ enables the protoplanet to overrun the gap and accret additional amount of mass. Mazeh et al. (1996) suggest that these companions may have $M_{p} \gg M_{J}$ and they belong to a brown dwarf population. This expectation is in contrast to the orbital properties of the eccentric companion of $16 \mathrm{Cyg}$ which has $P_{p}$ and $M_{p} \sin i$ smaller than those of 47 UMa's planetary companion. The latter has a nearly circular orbit. 
Numerical simulations indicate that although some planets may be ejected from the system, a residual population of eccentric planets may remain bound to the central star at large distances $(a>100 \mathrm{AU})$. Close encounters also excite the planets' inclination such that $i$ (in radian units) between the inner massive and outer eccentric planets is $\sim e / 2$. A search for additional planets around those stars with eccentric planets can provide important tests between these confronting models. Another useful test is the search for protoplanets around young stellar objects. In the gap overrun scenario, the massive eccentric planets are formed before the depletion of the disk gas and they are expected to be present among WTTS. But in the merger scenario, they are formed long after the gas removal such that systems of intermediate-mass protoplanets with modest separation and nearly circular orbits are expected to be found among WTTS.

The orbital instability scenario for the eccentric planets is analogous to the suggestion by Rasio et al. (1996) that the short period planets were dynamically scattered into a region near the surface of the central star (Ford \& Rasio 1996). Although $e$ of the short-period planets may be damped out, numerical simulations indicate that the scattering scenario would lead to the predication of a population of highly eccentric planets at large distances (10-100 AU) from the central stars. If the short-period planets were brought to the stellar proximity by the disk-planet interaction, additional planets far from the central stars may have nearly circular orbits. A more comprehensive search for these planets would provide a useful test for these competing scenarios.

\section{Unification scheme}

We suggest that the orbital properties of three types of planets are primarily determined by the mass distribution in the disk. Several cores of type (III) planets may be formed in the most massive disks. Since $T_{c}$ (and therefore $H$ ) increases with $\Sigma$ (Lin \& Papaloizou 1980), the protoplanet's truncation masses $\left(M_{f}\right)$ in high-mass disks are relatively high. Provided the ratio of $a$ between a pair of neighboring planets is not too large, the outer/inner Lindblad resonances of the inner/outer planet overlap with each other such that gas in the region between them is destabilized (Lin \& Papaloizou 1993) and ejected either interior to the orbit of the innermost planet or beyond the orbit of the outermost planet. The residual gas exterior to the planetary system may be inadequate to promote the radial migration of all the planets formed in these systems.

The intermediate-mass disks provide the nurseries for type (II) planets. In these disks, a smaller number of cores may be formed. Since both $T_{c}$ and $H$ have modest values, the protoplanets' $M_{f}$ may be $<M_{d}$ such that their orbital evolution is coupled to the viscous diffusion of the disk gas. If the first cores to acquire $M_{\text {crit }}$ emerge interior to $r_{\nu}$, they would migrate toward the host star. Additional protoplanets may be formed out of the residual gas in the intermediate and outer regions of the disk and they continue to migrate from their birth sites toward the host star (or outward in the outermost regions of the disk) until the disk mass is depleted to the limit that the residual gas can no longer induce any significant evolution of the protoplanets' orbit.

Type (I) planets are formed in low-mass disks which do not contain a sufficient mass to induce planetary orbital migration. It is also possible that type (I) planets are the survivors of the orbital evolution of type (II) planets. Low-mass disks similar to the solar nebula may once have had a mass similar to those 
which led to the formation and inward migration of several type (II) planets. The host stars of type (I) planets may have had a weak magnetic field during the their pre-main sequence evolution such that they were unable to prevent the migrating planets from merging into them in a manner analogous to type (II) planets. As the first born protoplanets perish, additional protoplanets form and migrate with the diffusion of the disk gas. When gas in the outer regions of the disk is depleted to $\Sigma \sim \Sigma_{\min }$, it can no longer induce any significant migration to the last batch of type (I) planets formed in such an environment.

This work is supported by NASA (NAGW-4967) and NSF (AST-9315578) grants.

\section{References}

Adams, F.C. Lada, C.J. Shu, F.H. 1987, ApJ312, 788

Artymowicz, P. \& Lubow, H. 1993, ApJ419, 166.

Artymowicz, P. \& Lubow, H. 1996, ApJ467, L77.

Beckwith, S.V.W. Sargent, A.I. Chini, R.S. Güsten R. 1990, AJ99, 924

Bodenheimer, P. \& Pollack, J.B. 1986, Icaurs, 67, 391

Bouvier, J. Cabrit, S. Fenandez, M., Martin, E., Mathews, J. 1993 A.Ap. 101,485

Butler, R. P. Marcy, G. W. ApJ, 458, 600

Butler, R. P. Marcy, G. W. \& Williams, 1996, ApJ, in press

Cameron, A.G.W. 1973, Icarus 18, 407

Cockran, W.D. Hatzes, A.P. Butler, R.P. Marcy, G.W. 1996, Science, in press

Chambers, J.E. Wetherill, G.B. Boss, A.P. 1996, Icarus, 119, 261

Goldreich P. Soter, S. 1966, Icarus 3, 375

Goldreich P. Tremaine, S. 1979 ApJ233, 857

Goldreich P. Tremaine, S. 1980 ApJ241, 425

Goodman, A.A. Benson, P.J. Fuller, G.A. Myers, P.C. 1993, ApJ, 406, 528

Franklin, F. Lecar, M. Quinn, T. 1990, Icarus 88, 97

Hartman, L. Kenyon, S. Hartigan, P. in Protostars and Planets III, eds. G.H. Levy, J.I. Lunine (Univ. Arizona Press: Tucson), 497

Hayashi, C. 1981, Prog.Theo.Phys.Suppl. 70, 35

Hayashi, C. Nakazawa, K., Nakagawa, Y. Protostars and Planets II, eds. D. Black, M. Mathews (Univ. Arizona Press: Tucson), 1100

Hayashi, M. Ohashi, N. Miyama, S.M. 1993, ApJ418, L71

Herbig, G. 1977 217, 693

Kley, W. Lin, D.N.C. 1996, ApJ461, 933

Konigl, A. 1991, ApJ370, L39

Latham, D. Mazeh, T. Stefanik, R. Mayor, M. Burki, G. 1989, Nature, 339, 38

Lada, C.J. Young, E.T. Greene, T.P. 1993, ApJ408, 471

Lay, O.P. Carlstrom, J. Hills, R.J. Phillips, T.G. 1994, ApJ, 434, L75

Lin, D.N.C. 1986, in The Solar System, ed M.G. Kivelson (Prentice-Hall), 28

Lin, D.N.C. 1996, in Molecular Clouds and Star Formation, eds C. Yuan J.H. You (World Scientific: Singapore), 261 
Lin, D.N.C. Bodenheimer, P.H. Richardson, D. 1996, Nature, 380, 607

Lin, D.N.C. Hayashi, M. Bell, K.R. Ohashi, N. 1994, ApJ435, 821

Lin, D.N.C. Ida, S. 1996, ApJin press

Lin, D.N.C. Papaloizou, J. 1986, ApJ309, 846

Lin, D.N.C. Papaloizou, J. 1993, in Protostars and Planets III, eds. G.H. Levy, J.I. Lunine (Univ. Arizona Press: Tucson), 749

Lissauer, J.J. 1987, Icarus, 69, 249

MacGregor, K.B. Brenner, M. 1991, ApJ376, 204

Marcy, G. W. \& Bulter, R. P. 1996, ApJ, 464, L147

Marcy, G. Bulter, R., Williams, E., Bildsten, L. \& Graham, J. 1996, preprint

Malhotra, R. 1995, AJ, 110, 420

Mayor, M. \& Queloz, D. 1995, Nature, 378, 355

Mazeh, T., Latham, D. Mayor, M. 1996, preprint

McCaughrean, M.J. Stauffer, J.R. 1994, AJ108, 1382

Mizuno, H. 1980, Prog. Theor. Phys. 64, 544

Mundy, L. Looney, L. Erickson, W. et al. 1996, ApJ464, L16

Palme H. \& Boynton, W.V. 1993 in Protostars and Planets III, eds. G.H. Levy, J.I. Lunine (Univ. Arizona Press: Tucson), 979

Papaliozou, J. Lin, D.N.C. 1984, 285, 818

Pollack, J.B., Hubickyj, O. Bodenheimer, P. Lissauer J.J. Podolak, M. Greenzweig, Y. 1996, Icarus, in press

Prosser, C.F. Stauffer, J.R. Hartmann, L. et al. 1994, ApJ421, 517

Rasio, F.A. Ford, E.B. 1996, Science, in press

Rasio, F.A. Tout, C.A. Lubow, S.H. Livio, M. 1996, ApJ, in press

Safronov, V. 1969, Evolution of the Protoplanetary Cloud and Formation of the Earth and Planets (Nauka: Moscow).

Skinner, S.L. Brown, A. Walter, F.M. 1991, AJ102, 1742

Strom, S.E. Edwards, S.E Skrutskie, M.F. 1993, in Protostars and Planets III, eds. G.H. Levy, J.I. Lunine (Univ. Arizona Press: Tucson), 837

Sargent, A.I. Beckwith, S. 1987, ApJ323, 294

Takeuchi, T. Miyama, S., Lin, D.N.C. 1996, ApJ460, 832

Tereby, S. Shu, F.H. Cassen, P. 1984, ApJ286, 529

Walter, F.M. Brown, A. Mathieu, R.D. Myers, P.C. 1988, AJ96, 297

Wetherill, G.W. Stewart, G.R. 1989, Icarus, 77, 330

Zuckerman, B. Forveille, T. Kastner, J.H. 1995, Nature, 373, 494

\section{Discussion}

M. Wardle: What proto-stellar disk masses were needed for you model?

D. Lin: A few $\%$ of $\mathrm{M}_{\odot}$. 